\title{
Schnellnachweis von Nitrit im Harn mittels Testpapier
}

\author{
Von \\ D. KUTTER \\ Aus dem Klinischen Laboratorium D. Kutter, Luxemburg
}

(Der Schriftleitung zugegangen am 9. November 1963)

\begin{abstract}
Die Herstellung eines haltbaren Testpapiers zum Nitritnachweis wird beschrieben. Die Methode stellt eine Abänderung der klassischen Reaktion nach Griess-Ilosvay dar. Auf die Hemmung der Reaktion durch Ascorbinsäure wird hingewiesen.
\end{abstract}

Preparation of a durable nitrite-test paper is described. The method is a modification of the classical GrIEss-ILOsVAY reaction. Inhibition of the reaction by ascorbic acid is reported.

Der Nitritnachweis sollte grundsätzlich bei jeder routinemäßigen Harnuntersuchung durchgeführt werden. Eine positive Nitritreaktion erlaubt nämlich nicht nur den eindeutigen Rückschluß auf das Vorhandensein von nitritbildenden Bakterien; in Gegenwart von Nitrit sind außerdem die EhrLICHsche Urobilinogenreaktion sowie der enzymatische Glucosenachweis mit Vorsicht $\mathrm{zu}$ bewerten. Es ist nämlich bekannt, daß diese Reaktionen durch Nitritionen gehemmt werden $(1,2)$.

Zum Nitritnachweis stehen eine Reihe von Reagenzien zur Verfügung. - Einen ersten Hinweis gibt eine positive Indolreaktion mit Sulfosalizylsäure (3), die man beim Eiweißnachweis beobachtet. Neben Nitrit $\mathrm{muß}$ jedoch auch bakteriengebildetes Indol vorliegen. Viel empfindlicher und sicherer ist das klassische Reagens nach Griess-Ilosvay, das wohl am meisten verwendet wird. Eine umfangreiche Arbeit über diese Reaktion und ihre Varianten wurde von WaLTHER $(4,5)$ veröffentlicht. - Außerdem hat sich die Nitrin-Reaktion nach Pfeiffer (6) bewährt.

Keines dieser Reagenzien stellt jedoch eine ausgesprochene Scbnellreaktion dar. Von den uns bekanntenen Varianten könnte das gut haltbare Trockenreagens nach JAEGGY und LANZ (7) als Schnelltest bezeichnet werden. Ein haltbares Testpapier erschien uns als eine noch handlichere Methode. Es erlaubt nicht nur die Ausführung der Reaktion am Krankenbett, sondern kann auch bei ambulanten Wasseruntersuchungen und Lebensmittelkontrollen ohne irgendwelche Hilfsmittel verwendet werden.

\section{Methodik}

Folgende Herstellungsweise ergab uns ein Testpapier, das uns in Haltbarkeit und Empfindlichkeit befriedigte:

$$
\begin{aligned}
& 6,2 \mathrm{~g} \alpha \text {-Naphtylamin } \\
& 1,0 \mathrm{~g} \text { Sulfanilsäure } \\
& 25 \mathrm{~g} \text { Citronensäure }
\end{aligned}
$$

werden im Mörser zerrieben und innig vermischt. Von diesem Pulver werden $0,5 \mathrm{~g}$ in $40 \mathrm{ml}$ Aqua dest. heiß gelöst. Mit der erkalteten Lösung werden Papierstreifen getränkt. Die Wahl des
Papiers ist besonders wichtig. Billige Filterpapiere enthalten stärkere Nitritspuren und ergeben eine lästige Grundfärbung. Mittelhartes Chromatographie-Papier (Arches) erwies sich uns als geeignet. Das Trocknen erfolgt am besten über konzentrierter Schwefelsäure im Vakuumexsikkator. Trocknen an freier Luft, sogar außerhalb des Laboratoriums ergab stets ein mehr oder weniger rosa gefärbtes Papier. Einmal getrocknet, ist das Testpapier unbegrenzt haltbar, solange es in einer gut schließenden Flasche mit etwas Silikagel aufbewahrt wird. Zum Gebrauch schneiden wir kleine Testblättchen von etwa $0,5 \mathrm{~cm} \times 0,5 \mathrm{~cm}$, die wir auf der FeIGLschen Tüpfelplatte verwenden. Es genügt, einen Tropfen Urin auf das Blättchen fallen zu lassen. Rosa- bis Rotfärbung ist für das Vorliegen von Nitritionen beweisend. Für ambulante Untersuchungen schneiden wird das Papier in schmale Streifen von etwa $0,3 \mathrm{~cm} \times 3 \mathrm{~cm}$. Ein solcher Streifen kann z. B. direkt in den Harnstrom gehalten werden. Der Reaktionsausfall ist der gleiche.

Als Empfindlichkeitsgrenze fanden wir $1 \mathrm{mg} \%$ Natriumnitrit in Wasser. $0,5 \mathrm{mg} \%$ sind eben noch wahrnehmbar. - Der Reaktionsmechanismus entspricht demjenigen der klassischen Methode nach GrIEss-ILOsvaY: Die salpetrige Säure bildet mit der Sulfanilsäure eine Diazoverbindung, die sich ihrerseits mit dem $\alpha$-Naphtylamin zu einem karminroten Azofarbstoff kuppelt. Das Testpapier ist demnach ebenso spezifisch wie die Originalmethode. Über etwaige Hemmungen der Reaktion konnten wir keinerlei Angaben finden. - Aus einer Arbeit von Zausch (8) ersahen wir, daß die Bilirubinbestimmung mit der Diazoreaktion durch Ascorbinsäure gehemmt wird. Wir haben daher die Möglichkeit einer solchen Hemmung auf unser Testpapier - und somit auf die Reaktion nach GrIessILOSVAY - untersucht.

Nitritfreien Harnen wurden steigende Mengen Natriumitrit und Ascorbinsäure zugesetzt. Die Untersuchung dieser Mischungen mit dem Testpapier ergab folgende Werte:

\begin{tabular}{cccccc}
\hline Zugefügte Ascorbinsäure mg\% & 0 & 50 & 200 & 500 \\
\hline Zugefügtes $\mathrm{NaNO}_{2} \mathrm{mg} \%$ & \multicolumn{2}{c}{ Reaktion des } & Testpapiers \\
1 & + & 0 & 0 & 0 \\
2 & ++ & $(+)$ & $(+)$ & 0 \\
5 & +++ & ++ & $(+)$ & 0 \\
\hline
\end{tabular}


Ascorbinsäure hat demnach auf diese Nachweisreaktion einen deutlichen Hemmeffekt. Bei der heute üblichen, besonders hohen Vitamin-C-Medikation sind Harnspiegel von $50 \mathrm{mg} \%$ und $\mathrm{mehr}$, keine Seltenheit. Bei festgestellter Ausscheidung von Ascorbinsäure ist eine negative Nitritreaktion demnach mit Vorsicht zu bewerten. Ein Abklingen der Nitritreaktion nach intensiver Vitaminbehandlung ist nicht unbedingt auf das
Verschwinden des nitritbildenden Erregers zurückzuführen. - Diese Hemmung kann sich möglicherweise beim Nitritnachweis im Hackfleisch nachteilig auswirken, da der Zusatz von Ascorbinsäure in vielen Ländern gestattet ist. Bei Vorhandensein von Ascorbinsäure ist eine negative GrIEss-Reaktion mit Vorsicht $z u$ bewerten. Über Untersuchungen zu diesem Problem werden wir anderwärtig berichten.

\section{Literatur}

1. Bolte, A. und A. Finke, Landarzt, Stuttgart 32, 350 (1956). 2. Kutrer, D., Bull. Soc. sc. med. Luxembourg 97, 259 (1960). - 3. Kutrer, D., Zschr. Urol. 54, 57 (1961). - 4. WALTHER, H. J., Aerztl. Laborat. 6, 287 (1960). - 5. Walther, H. J., Aerztl.
Laborat. 9, 272 (1963). - 6. Pfeiffer, H., Münch. med. Wschr. 92, 1315 (1950). - 7. JaEGGY, E. und W. LANZ, Schweiz. med. Wschr. 65, 363 (1935). - 8. ZAusch, G., Aerztl. Laborat 2, 137 (1956).
Dr. rer. nat. Dolphe Kutter Klinisches Laboratorium 14, rue Beck Luxembourg

\section{Buchbesprechungen}

Vitamin-Bestimmungen. Herausgegeben von E. Merck AG., Darmstadt. Von R. Strohecker und H.M. Henning. $365 \mathrm{~S}$, 42 Abb., 8 Farbtafeln, DM 42,-. Verlag Chemie, GmbH., Weinheim/Bergstr. 1963.

Mit der immer größer werdenden Anwendung der Vitamine in der Arzneimittel-, Lebensmittel- und Futtermittelindustrie steigt das Interesse an der quantitativen Erfassung dieser Wirkstoffe. Das Vitamin-Untersuchungslaboratorium der Chemischen Fabrik Merck in Darmstadt gab schon vor Jahren eine Zusammenstellung von chemischen und physikalisch-chemischen Vitaminbestim- mungsmethoden heraus, die sich.schnell großer Beliebtheit erfreute und bald vergriffen war. Unter der Autorschaft der Herren Strohecker und Henning ist nun die neue Monographie „Vitamin-Bestimmungen, exprobte Methoden" herausgebracht worden, der zweifellos eine gute Prognose gestellt werden kann. Man spürt bei der Darstellung die kundigen Analytiker, die mit didaktischem Geschick und, unterstützt durch zahlreiche - auch z. T. bunte - Abbildungen, die verschiedenen Methoden beschreiben.

R. Ammon, Homburg/Saar

\section{Termine}

24. - 25. 4. 1964

Gent, 4th West-European Symposium on Clinical Chemistry

3rd Symposium on Enzymes in Clinical Chemistry.
20.-24. 5. 1964

26. 7.-1. 8. 1964

22. -24.10 .1964$
Eindhoven, Kongreß der Königlich Niederländischen Chemischen Vereinigung

New York, 6th International Congress of Biochemistry.

Köln, Kongreß für Physiologische Chemie.

Die Wiedergabe von Gebraucbsnamen, Handelsnamen, Warenbezeicbnungen und dgl. in dieser Zeitscbrift berecbtigt nicbt zu der Annabme, daß solebe Namen obne weiteres von jedermann benülzt werden dürfien. Vielmebr bandelt es sicb bäufig um gesetzlicb gescbützte Warenzeicben, aucb vonn sie nicbt eigens als solcbe gekennzeicbnet sind.

Verlag Walter de Gruyter \& Co., vormals G. J. Göschen'sche Verlagshandlung · J. Guttentag, Verlagsbuchhandlung · Georg Reimer · Katl J. Trübner · Veit \& Comp., 1 Berlin 30, Genthiner Str. 13; 1964. - Printed in Germany. - Satz und Druck: Walter de Gruyter \& Co., 1 Berlin 30, Genthiner Str. 13. - Anzeigen: Merkur-Werbung, Dr. K. Jeserich KG, 7 Stuttgart 1, Postfach 740, Tel. 246358/59/50. - Für den Anzeigenteil verantwortlich: Willibald Plitzko. 